\title{
COORDENAÇÃO VERTICAL E CONTRATOS INFORMAIS NA AGRICULTURA IRRIGADA: UM ESTUDO DE CASO COM APLICAÇÃO DO MODELO TOBIT ${ }^{1}$
}

\author{
Ricardo Chaves Lima ${ }^{2}$ \\ José Ferreira Irmão ${ }^{3}$
}

Resumo - A teoria dos contratos parte do pressuposto que a natureza humana é fundamentalmente oportunista e que os agentes econômicos devem proteger-se da seleção adversa (adverse selection) e dos perigos relacionados com conduta moral (moral hazards) pela elaboração de contratos. No caso das áreas irrigadas no Nordeste, esses contratos podem ser formais ou informais. Este trabalho parte do pressuposto que a realização de contratos entre produtores de acerola e a agroindústria, no Pólo de Irrigação Petrolina-Juazeiro, depende de características socioeconômicas dos produtores e de fatores relacionados com unidades produtivas. $\mathrm{O}$ modelo empírico utilizado foi o tobit, o que é justificado pela ocorrência de variável dependente com distribuição truncada na regressão. Os resultados mostram que a decisão de engajar-se em uma relação contratual é definida pelo grau de dependência do agricultor para com a agroindústria e pelas experiências passadas sobre realização de contratos.

Palavras-chave: coordenação vertical, contratos, irrigação, modelo tobit.

\section{Introdução}

O pólo de irrigação Petrolina-Juazeiro conta hoje com, aproximadamente, 100.000 ha de área irrigada, onde são produzidos produtos agrícolas para comercialização in natura e também insumos para a agroindústria. Existe, portanto, uma cadeia produtiva com elos que se articulam a "montante" e a "jusante". Essa articulação faz-se por meio de contratos que podem ser formais ou informais. Os informais, no entanto, ocorrem com

\footnotetext{
${ }^{1}$ Recebido em 02/03/2004. Aceito em 25/03/2004.

${ }^{2}$ Professor do Departamento de Economia/PIMES - UFPE // Av. dos Economistas s/n, Cidade Universitária, Recife-PE.E-mail: rlima@ufpe.br.

${ }^{3}$ Professor do Departamento de Letras e Ciências Humanas - UFRPE. E-mail: jose @ hotlink.com.br
} 
mais freqüência no perímetro irrigado Petrolina-Juazeiro (Lima e FerreiraIrmão, 2002).

A discussão sobre contratos, originariamente desenvolvida pela teoria da organização industrial, é de grande relevância para o agronegócio. A teoria dos contratos parte do pressuposto que a natureza humana seja fundamentalmente oportunista e que os agentes econômicos devam proteger-se da seleção adversa (adverse selection) e dos perigos relacionados com conduta moral (moral hazards) pela elaboração de contratos (Lyons, 1994). A relação contratual na agroindústria irrigada do Nordeste varia desde a informalidade da intenção de entrega até a formalização de um documento contratual.

Neste trabalho examinam-se os fatores que levam os agentes econômicos na cadeia produtiva a engajar-se em uma relação contratual, embora sem a formalização de um documento escrito. Os dados são referentes à pesquisa de campo realizada entre os produtores de acerola no Pólo de Irrigação Petrolina-Juazeiro, no ano de 1999. O modelo empírico utilizado foi o tobit, o que é justificado pela ocorrência de variável dependente com distribuição truncada na regressão.

\section{O pólo de irrigação Petrolina-Juazeiro}

O desenvolvimento da agricultura irrigada no semi-árido nordestino tem se deparado com entraves, dada a falta de recursos para utilização das tecnologias disponíveis para aproveitamento das fontes de água existentes. Algumas áreas na região apresentam potencial para desenvolvimento da agricultura irrigada, em razão de sua proximidade com fontes perenes de água de boa qualidade para irrigação. Esse é o caso, por exemplo, da região do Vale do São Francisco, onde se têm identificado grandes áreas propícias ao desenvolvimento da agricultura irrigada.

As políticas de desenvolvimento do Vale do São Francisco têm apoiado, de forma prioritária, o desenvolvimento da agricultura irrigada. A maior 
concentração de áreas irrigadas encontra-se no submédio São Francisco. Essa região, que corresponde a $66 \%$ da área total do vale, tem sido beneficiada por investimentos em infra-estrutura de irrigação, o que tornou possível o desenvolvimento de uma agricultura caracterizada pela utilização de tecnologias de produção intensivas em capital e de elevada produtividade (Ferreira Irmão, 1995).

O pólo de irrigação de maior importância na região do submédio São Francisco é o de Petrolina-Juazeiro. Esse pólo compreende seis projetos públicos de irrigação, implantados pela CODEVASF; dois deles localizados no lado pernambucano (Bebedouro e Nilo Coelho) e quatro no lado baiano (Curaça, Mandacaru, Maniçoba, Tourão). Foram também implantados na região, pela CHESF, três projetos; dois no lado pernambucano (Caraíbas e Brígida) e um no lado baiano (Pedra Branca). O projeto Nilo Coelho é o que apresenta a maior área irrigada e também a maior área de expansão. O referido projeto compreende uma área de 22.130,31 ha, destinada às firmas, e de 16.638,00 ha, destinada ao assentamento de colonos.

Os projetos de irrigação do pólo Petrolina-Juazeiro produzem frutas e culturas hortícolas. As frutas correspondem a cerca de $33 \%$ da área plantada, enquanto as demais culturas correspondem ao restante da área. Entre as frutícolas, os principais cultivos são de uva, banana, manga, melancia, melão e acerola, os quais correspondem a cerca de $80 \%$ do valor comercializado das frutas. As frutas correspondem também a 40\% do valor dos produtos agrícolas comercializados no pólo (Lima e FerreiraIrmão, 2002).

A produção agrícola no pólo é destinada, em parte, ao consumo in natura, sendo exportada para outras regiões do Brasil e para fora do país. Outra parte da produção é beneficiada por empresas processadoras que foram instaladas na região a partir dos anos setenta. Como exemplo dessas indústrias, podem-se citar as processadoras de tomate, que produzem extrato e molho de tomate. Foram também instaladas na região indústrias processadoras de vinho e conserva de frutas, e usinas beneficiadoras de açúcar. A instalação dessas indústrias no pólo Petrolina-Juazeiro tem 
sido considerada importante para o desenvolvimento, visto que é responsável pela geração de emprego e pela agregação de valor ao produto agrícola local.

O grande número de produtores agrícolas e o pequeno número de empresas processadoras estimulam um ambiente de competição imperfeita entre esses agentes econômicos, ou seja, pode-se supor que haja tendência de atuação das empresas processadoras como monopsônios. Esse poder de mercado das processadoras é tanto maior quanto menor é a possibilidade de mercados alternativos para os produtores agrícolas. Espera-se, também, que outros fatores, como nível tecnológico da produção, custos de transação e características socioeconômicas dos agricultores, influenciem o relacionamento entre a agricultura e a indústria. $\mathrm{O}$ problema de pesquisa, considerado neste trabalho, pode ser resumido na seguinte pergunta: Quais os fatores que influenciam a coordenação vertical entre os produtores agrícolas e a indústria e qual a importância relativa desses fatores?

\subsection{Custos de transação e teoria dos contratos}

A economia dos custos de transação discute a questão da organização econômica como um problema contratual (Williamson, 1996). A Tabela 1 descreve os atributos do processo de contratos em uma cadeia produtiva. A suposição comportamental é a de que os indivíduos têm racionalidade limitada, ou seja, têm informações limitadas, e, ou, podem assumir postura oportunista. Essa suposição será assumida na presença ou ausência de ativos específicos. ${ }^{4}$ Neste estudo, os contratos são relacionados com: (i) planejamento, (ii) promessa, (iii) competição e (iv) governança, dependendo da suposição comportamental pertinente à troca e aos atributos econômicos dos bens e serviços em questão (Williamson, 1985). Considere que cada uma dessas condições possa ser representada pelo sinal (+), quando presente, e por (-), quando ausente.

\footnotetext{
${ }^{4}$ Entende-se por ativos específicos aqueles que apresentam poucos usos alternativos.
} 
Tabela 1 - Atributos do processo de contrato

\begin{tabular}{|c|c|c|c|}
\hline \multicolumn{2}{|c|}{ Suposição comportamental } & \multirow{2}{*}{$\begin{array}{c}\text { Especificidade } \\
\text { dos ativos }\end{array}$} & \multirow{2}{*}{$\begin{array}{c}\text { Processo } \\
\text { contratual } \\
\text { pertinente }\end{array}$} \\
\hline $\begin{array}{c}\text { Racionalidade } \\
\text { limitada }\end{array}$ & Oportunismo & & \\
\hline- & + & + & Planejamento \\
\hline+ & - & + & Promessa \\
\hline+ & + & - & Competição \\
\hline+ & + & + & Governança \\
\hline
\end{tabular}

Fonte: Adaptado de Williamson (1985).

No primeiro caso, os agentes econômicos são oportunistas, os ativos são específicos, mas a racionalidade não é limitada. Assim, os agentes têm informações suficientes para engajar-se em um processo pormenorizado de barganha desde o início da negociação. O processo contratual pertinente, portanto, é o de planejamento.

O segundo caso refere-se à ocorrência de racionalidade limitada, especificidades dos ativos, mas ausência de oportunismo. Nesse caso, é suficiente a palavra dos agentes na realização dos contratos. O processo contratual pertinente, portanto, é o de promessa.

No terceiro caso, têm-se racionalidade limitada e oportunismo, mas os ativos não são específicos, ou seja, na ausência de especificidade dos ativos, as partes engajadas em uma possível relação de troca não têm interesse mútuo em uma relação de negociação ex ante. Assim, o processo contratual pertinente é o de competição.

No último caso, têm-se racionalidade limitada, oportunismo dos agentes e especificidade dos ativos. O planejamento é incompleto, por falta de informações; as promessas não são confiáveis, por falta de credibilidade; e os agentes têm maior identidade de relacionamento, por estarem lidando com ativos específicos. Assim, o processo contratual pertinente é o de governança. 
Estudos empíricos têm concentrado atenção em contratos formais entre empresas que se relacionam em uma cadeia produtiva. Lyons(1994) examinou os determinantes da probabilidade de os agentes escreverem um documento formal que regule a relação contratual entre eles. Entre os fatores relacionados no estudo estão:

- Vulnerabilidade: diz respeito à vulnerabilidade de um agente à conduta oportunista do outro. Quando, por exemplo, a maior parte da produção de um dos agentes é especializada para um cliente em particular, este agente deve ter maior preocupação com a formalização de um contrato. Essa preocupação, no entanto, deve ser menor quando o produto especializado for apenas uma fração pequena do produto total. Assim, espera-se que o aumento da vulnerabilidade eleve a probabilidade de se realizar um contrato.

- Complexidade: os contratos serão fáceis de escrever, se o produto for simples e imutável. Produtos complexos tornam os contratos complicados e requerem a necessidade de contratar consultores especialistas para escrevê-los. Assim, espera-se que o aumento da complexidade reduza a propensão a escrever contratos.

- Tamanho: quanto maior for a escala de produção das firmas envolvidas, maiores serão as conseqüências de perdas por causa de um contrato mal feito ou pouco específico. Assim, espera-se que a probabilidade de se estabelecer uma relação contratual aumente com o tamanho da firma.

- Confiança: um dos motivos da relação contratual é a falta de confiança entre as partes. Assim, espera-se que, quanto maior for o nível de confiança, menor será a necessidade de relações contratuais mais rígidas.

Utilizando-se a Tabela 1 como referencial analítico, pode-se supor que a diferença fundamental entre contratos formais e informais seja a ocorrência de agentes oportunistas. Assim, um contrato informal, na ausência de oportunismo, teria efeito semelhante a um contrato formal. Nesse 
caso, variáveis relacionadas com esses fatores acima também poderiam influenciar a realização de um contrato informal. A alternativa ao contrato informal seria a não realização de contratos.

\subsection{Dados da pesquisa}

Os dados utilizados neste estudo foram coletados em pesquisa de campo, realizada entre janeiro e março do ano de 1999, no Pólo de Irrigação Petrolina-Juazeiro, estado de Pernambuco. A amostra utilizável inclui 75 observações, retiradas aleatoriamente entre os produtores de acerola no perímetro de irrigação. Em cada lote, os produtores estudados apresentaram uma área total irrigada e uma fração desta como área contratada com a agroindústria para entrega da produção. Assim, foi calculado um índice de coordenação vertical agricultura-indústria, que é igual à área contratada dividida pela área total irrigada. Esse índice variou de zero a um, ou seja, entre agricultores que decidiram pelo não engajamento em relações contratuais até aqueles que decidiram contratar toda a área irrigada. Foram coletadas também informações socioeconômicas e de produção entre os produtores.

\section{Modelo empírico}

O modelo tobit é utilizado nos casos em que a variável dependente apresenta concentração de pontos com valores iguais a um valor limite, geralmente zero. O modelo estocástico subjacente é apresentado a seguir:

$$
\begin{array}{cc}
y_{t}=X_{t} \alpha+\mu_{t} & \text { se } X_{t} \alpha+\mu_{t}>0 \\
=0 & \text { se } X_{t} \alpha+\mu_{t} \leq 0 \\
& t=1,2, \ldots N
\end{array}
$$


em que $N$ é o número de observações; $y_{t}$, variável dependente; $X_{t}$, vetor de variáveis independentes; $\alpha$, vetor de coeficientes desconhecidos; e $\mu_{t}$ erro aleatório, que é distribuído com média 0 e variância constante (McDolnald e Moffitt, 1980).

Um índice I é criado como uma função linear das variáveis independentes. Esse índice, segundo White (1993), pode ser escrito como segue:

$$
I_{t}=X_{t} \alpha=X_{t}(\beta / \sigma) \text {. }
$$

Os estimadores do modelo tobit são o parâmetro de normalização $\sigma$ e o vetor $\alpha$, de coeficientes normalizados. $\mathrm{O}$ índice $I$ é transformado em uma variável dependente limitada, estimada pelas funções de densidade normal e normal cumulativa.

Admitindo-se que o valor limite seja zero, as observações da variável dependente podem ser ordenadas, tal que as $S$ primeiras observações tenham $y_{t}=0$ e as últimas $N$-S observações tenham $y_{t}>0$, os parâmetros $\alpha$ e $\sigma$ podem ser estimados pela maximização do valor da função logverossimilhança, como descrito a seguir:

$$
\left.L=\sum_{t=1}^{S} \log \left[1-F\left(\sigma y_{t}-I_{t}\right)\right]+\sum_{t=S+1}^{N} \log f\left(\sigma y_{t}-I_{t}\right)\right]
$$

Os coeficientes normalizados estimados do modelo tobit não podem ser usados diretamente para análise dos impactos das variáveis independentes na variável dependente, visto que esses coeficientes representam impactos, considerando-se as transformações das funções de densidade normal. É possível, no entanto, calcular um efeito marginal dos coeficientes, o que torna a interpretação semelhante aos coeficientes produzidos pelo método dos mínimos quadrados. Na análise feita neste trabalho serão consideradas a direção dos impactos (sinais) e as relativas magnitudes dos coeficientes, o que dispensa o cálculo dos efeitos marginais. As variáveis a serem utilizadas no modelo empírico são descritas na Tabela 2. 
Tabela 2 - Descrição das variáveis

\begin{tabular}{|c|c|}
\hline Variáveis & Descrição \\
\hline ICV & $\begin{array}{l}\text { Índice de coordenação vertical em área irrigada/área } \\
\text { total (variável dependente) }\end{array}$ \\
\hline IDADE & Idade do(a) chefe da família (anos) \\
\hline EDU & $\begin{array}{l}\text { Média de educação da família (anos de educação } \\
\text { formal) }\end{array}$ \\
\hline ECIVIL & $\begin{array}{l}\text { Estado civil do(a) chefe da família ( } 1=\text { casado/ } \\
\text { amasiado, } 0=\text { caso contrário) }\end{array}$ \\
\hline MOFT & $\begin{array}{l}\text { Mão-de-obra familiar que trabalha na propriedade } \\
\text { (número de pessoas) }\end{array}$ \\
\hline MOFTF & $\begin{array}{l}\text { Mão-de-obra familiar que trabalha fora da proprie- } \\
\text { dade (número de pessoas) }\end{array}$ \\
\hline VISITA & $\begin{array}{l}\text { Visitas realizadas pela agroindústria à propriedade } \\
\text { (número de visitas por ano) }\end{array}$ \\
\hline VISITA3D & $\begin{array}{l}\text { Visitas realizadas pelo terceiro distrito à propriedade } \\
\text { (número de visitas por ano)* }\end{array}$ \\
\hline INSUMO & $\begin{array}{l}\text { Variável binária ( } 1=\text { recebe insumos da agroindústria, } \\
0=\text { caso contrário) }\end{array}$ \\
\hline CONTRATO & $\begin{array}{l}\text { Variável binária }(1=\text { se realizou contrato no passado, } \\
0=\text { caso contrário) }\end{array}$ \\
\hline AREAPT & Área plantada total (em hectares) \\
\hline EXP & $\begin{array}{l}\text { Experiência como produtor no perímetro irrigado } \\
\text { (anos) }\end{array}$ \\
\hline
\end{tabular}

*O terceiro distrito é uma organização de gestão do perímetro irrigado Nilo Coelho, ligado à CODEVASF. 


\section{Resultados}

Os coeficientes do modelo empírico estimado são apresentados na Tabela 3. Foram estimados 2 modelos. O primeiro inclui, predominantemente, as variáveis relacionadas com produção, enquanto o segundo inclui, predominantemente, as variáveis relacionadas com perfil demográfico do produtor.

Tabela 3 - Resultados das estimações do modelo tobit

\begin{tabular}{lcc}
\hline \multirow{2}{*}{ Variáveis } & \multicolumn{2}{c}{ Coeficientes normalizados } \\
\cline { 2 - 3 } IDADE & $\ldots$ & Modelo 1 \\
EDU & $\ldots$ & $-0,0602(-3,573)^{*}$ \\
ECIVIL & $\ldots$ & $0,0537(1,056)$ \\
MOFT & $-0,1429(-1,980)^{*}$ & $-0,1573(-0,375)$ \\
MOFTF & $\ldots$ & $\ldots$ \\
VISITA & $0,9428(1,963)^{*}$ & $0,3466(2,611)^{*}$ \\
VISITA3D & $-0,0026(0,006)$ & $\ldots$ \\
INSUMO & $0,9889(3,268)^{*}$ & $\ldots$ \\
CONTRATO & $-1,1189(-2,727)^{*}$ & $\ldots$ \\
AREAPT & $0,7116(5,868)^{*}$ & $0,5227(4,264)^{*}$ \\
EXP & $-0,1430(-3,020)^{*}$ & $-0,1576(-3,039)^{*}$ \\
\hline
\end{tabular}

Nota: os números entre parênteses correspondem à estatística $t$, de student. *estatisticamente significante a $5 \%$. 
Entre as variáveis relacionadas com perfil demográfico do produtor, a única que apresentou coeficiente estatisticamente significante foi IDADE. O sinal negativo desse coeficiente indica que a propensão à realização de contratos diminui com o aumento da idade. Esse padrão pode estar relacionado com a falta de costume dos produtores mais velhos na realização de contratos, já que uma parte dos produtores do perímetro veio da agricultura de subsistência. É possível também que a variável idade esteja relacionada com experiências passadas com contratos.

As variáveis MOFT e MOFTF tiveram coeficientes estatisticamente significantes. Os sinais positivo de MOFTF e negativo de MOFT sugerem que o emprego da mão-de-obra familiar fora da fazenda possa estar relacionado com menor propensão ao engajamento em uma relação contratual. Na relação contratual típica, a agroindústria normalmente entra com uma contrapartida de insumos, como horas de máquinas, o que pode estar relacionado com liberação de mão-de-obra para fora da fazenda.

A variável VISITA tem coeficiente positivo e estatisticamente significante, o que sugere que as visitas técnicas da agroindústria aumentem a propensão dos produtores ao engajamento em relações contratuais. Essa variável pode estar ligada ao fator vulnerabilidade, ou seja, as visitas técnicas da agroindústria deixariam os produtores mais dependentes tecnologicamente. No caso das visitas técnicas do terceiro distrito (VISITA3D), o sinal negativo do coeficiente é o esperado, já que maior relacionamento com o terceiro distrito deixa os produtores menos dependentes da agroindústria. O coeficiente estimado dessa variável, no entanto, não é estatisticamente significante. A variável INSUMO teve coeficiente estimado positivo e estatisticamente significante. Esta variável segue a mesma lógica da variável VISITA, ou seja, o recebimento de insumos da agroindústria torna os agricultores mais dependentes e, portanto, mais propensos à realização de contratos.

A variável CONTRATO mostrou coeficiente negativo e estatisticamente significante. Considerando-se que ela represente experiências passadas com a coordenação vertical no perímetro irrigado, pode-se entender que esse resultado sugere que a relação contratual tem sido uma experi- 
ência negativa para os produtores. A variável EXP, que representa a experiência do produtor no perímetro irrigado, também mostrou coeficiente estimado estatisticamente significante e negativo. Este resultado pode indicar que o conhecimento do produtor, no que se refere às relações contratuais com a indústria, por experiência própria ou não, influenciou negativamente a decisão de realizar contratos com a agroindústria. A variável AREAPT, que representa a área total plantada, teve coeficiente estimado estatisticamente significante e com sinal positivo, o que sugere que um aumento da área plantada eleve a propensão a engajarse em relação contratual.

\section{Conclusões e comentários finais}

Os produtores no perímetro irrigado têm a opção de produzir para o mercado após a colheita, produzir para a agroindústria sem contrato ou produzir para a indústria de acordo com um contrato prévio. Os resultados deste trabalho mostram que a decisão de engajar-se em uma relação contratual é fundamentalmente definida pelo grau de dependência que o agricultor tem da agroindústria e das experiências passadas com relação à realização de contratos. De acordo com os resultados obtidos, os agricultores desenvolvem suas estratégias de maximização de retornos com base nas atividades agrícolas e no emprego da mão-de-obra fora da fazenda. Assim, a diminuição do risco de mercado com o engajamento em contrato reduz as expectativas de retorno devido aos preços mais baixos pagos pela agroindústria, mas libera mão-de-obra para o emprego fora da fazenda.

Outro aspecto que vale a pena mencionar é que a organização dos produtores em associações ou cooperativas pode ser de grande importância no poder de barganha do produtor com a agroindústria. Apesar de a relação entre as visitas técnicas do terceiro distrito às fazendas e de o índice de integração não terem mostrado coeficiente estimado estatisticamente significante, o sinal negativo pode ser um indício de que o poder de barganha dos agricultores aumenta quando diminui a dependência técnica desses para com a indústria processadora. 
Uma limitação deste trabalho é a inclusão de apenas uma cultura, no caso a acerola, na amostra. É importante, portanto, examinar outras culturas que apresentem níveis distintos de especificidade de ativos, com vistas em examinar o impacto desse fator na propensão à realização de contratos. Seria também estimulante examinar, tal como em Lyons (1994), os fatores que determinam a realização de contratos formais.

\section{Referências bibliográficas}

FERREIRA IRMÃO, J. 1995. “Agricultura irrigada e produção para exportação no vale do São Francisco." Texto Para Discussão No. 358, PIME/UFPE.

LIMA, R.C e Ferreira-Irmão, J. Um exame dos determinantes da coordenação vertical no pólo de irrigação Juazeiro-Petrolina. Relatório de Pesquisa. Recife, 2002.

LYONS, B. R. Contracts and specific investments: an empirical test of transaction cost theory. Journal of Economics and Management Strategy, Vol. 3, No. 2. 1994

MCDONALD, J. F. e Moffitt, R. A. The uses of tobit analysis. The Review of Economics and Statistics. 62, 1980.

WHITE, K. J. Shazam User's Reference Manual Version 7.0. McGraw-Hill, 1993.

WILLIAMSON, O. E. The Mechanisms of Governance. Oxford University Press, 1996.

WILLIAMSON, O. E. The Economic Institutions of Capitalism. Free Press, New York, 1996. 
Abstract - The theory of contracts assumes that human nature is essentially opportunistic, and economic agents protect themselves against adverse selection and moral hazard by elaborating contracts. In the case of irrigated areas in the Brazilian northeast, such contracts are not necessarily formal. That is, arrangements based on economic agent's word are common. The data used are from acerola producers in the Petrolina-Juazeiro irrigation project. The empirical model was tobit, given that the dependent variable was characterized by a truncated distribution. The results showed that the decision to engage on contractual relations is fundamentally defined by the degree of dependence of the farmer in respect to the processing industry. Furthermore, past experience of contractual relationship with the processing industry is a strong determinant of the individual's behavior.

Key-words: vertical coordination, contracts, irrigation, tobit model. 